Canadian

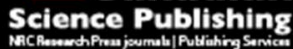

Canadian Geotechnical Journal Revue canadienne de géotechnique

\title{
A water retention model for compacted bentonites
}

\begin{tabular}{|r|l|}
\hline Journal: & Canadian Geotechnical Journal \\
\hline Manuscript ID & cgj-2016-0297.R2 \\
\hline Manuscript Type: & Article \\
\hline Date Submitted by the Author: & $28-$ Dec-2016 \\
\hline Complete List of Authors: & $\begin{array}{l}\text { Dieudonne, Anne-Catherine; Université de Liège (ULg) } \\
\text { Della Vecchia, Gabriele; Politecnico di Milano } \\
\text { Charlier, Robert; Université de Liège (ULg) }\end{array}$ \\
\hline Keyword: & $\begin{array}{l}\text { Water retention model, Compacted bentonites, Unsaturated soils, } \\
\text { Microstructure }\end{array}$ \\
\hline
\end{tabular}




\title{
A water retention model for compacted bentonites
}

\author{
Anne-Catherine Dieudonne*1, Gabriele Della Vecchia ${ }^{2}$, and Robert \\ Charlier $^{1}$ \\ ${ }^{1}$ University of Liege, Belgium \\ ${ }^{2}$ Politecnico di Milano, Italy
}

December, 2016

\begin{abstract}
The water retention behaviour of compacted bentonites is strongly affected by multi-physical and multi-scale processes taking place in these materials. Experimental data have evidenced major effects of the material dry density, the imposed volume constraints and the soil fabric. This paper presents a new water retention model accounting for proper retention mechanisms in each structural level of compacted bentonites, namely adsorption in the intra-aggregate pores and capillarity in the inter-aggregate ones. The model is calibrated and validated against experimental data on different bentonite-based materials, showing good capabilities in capturing the main features of the behaviour. The model is able to reproduce experimental data on compacted bentonites over a wide range of suction values, within a unified framework and using a limited number of parameters. Some of the parameters introduced are shown to take approximately the same value for several bentonites, providing a significant basis for preliminary design when dedicated experiments are missing.
\end{abstract}

\footnotetext{
*anne-catherine.dieudonne@epfl.ch
} 


\section{${ }_{20}$ Introduction}

The water retention curve is one of the fundamental properties required for predicting the behaviour of unsaturated soils. Early water retention models (Gardner 1958; Brooks and Corey 1964; van Genuchten 1980; Fredlund and Xing 1994) were formulated as a unique relationship between suction and the degree of saturation (volume of water over volume of voids) or the water content (mass of water over solid mass). These models were later extended to account for the influence of void ratio on the water retention curve and more specifically, on the air-entry value (see Gallipoli et al. 2003; Mbonimpa et al. 2006; Nuth and Laloui 2008; Tarantino 2009; Masin 2010; Zhou et al. 2012, among others).

In the case of compacted clays, the aggregated fabric of the materials, characterized by intra-aggregate and inter-aggregate pores, has been recognized to play a significant role on the water retention properties. In particular, the influence of clay fabric and its evolution have been discussed in the literature highlighting the role of compaction water content (Thom et al. 2007), the hydro-mechanical path (Lloret et al. 2003; Romero et al. 2011; Dieudonne et al. 2014a; Seiphoori et al. 2014; Della Vecchia et al. 2015) and the chemical composition of the pore fluid (Mata et al. 2002; He et al. 2016; Thyagaraj and Salini 2016). Accordingly, in recent years, water retention models have been developed to account for the role of the compacted clay fabric. These models mainly rely on information provided by mercury intrusion porosimetry (MIP), linking the cumulative pore volume to an apparent pore size. MIP results do not only provide useful information about the pore size distribution of a porous medium, but they are also exploited to obtain information about the water retention properties via the Laplace equation, which establishes a relation between the pore radius and the matric suction. Romero and Vaunat (2000) proposed the first water retention model accounting for different retention mechanisms in the different structural domains, introducing void ratio dependency only at low suction level, where capillarity phenomena dominates. The same approach was followed by Romero 
et al. (2011) and Della Vecchia et al. (2013), where the role on the hydraulic response of compacted clay of the evolution of aggregate size has also been introduced. MIP information has also been explicitly introduced in water retention modelling by other Authors ful 2002; Casini et al. 2012; Beckett and Augarde 2013; Hu et al. 2013; Arroyo et al. 2015).

Despite providing satisfactory results for medium to low activity clay materials, the models proposed up to now are not particularly suited to simulate the retention properties 55 swells significantly upon wetting, resulting in important changes in dry density that are not easily accounted for by the existing water retention models. For these materials swelling is mainly related to the significant volume changes of the aggregates upon hydration and the volume of water held by the microstructure is in several applications significantly stored by adsorption, it is evident that the capillary tube scheme is not representative of the physical processes taking place in such materials and that more realistic physical processes have to be considered to overcome pure phenomenological approaches. Another significant drawback of the current modelling approaches for the water retention behaviour the models. Calibration procedures are often heavy, requiring information to be collected at both the laboratory and microscopic scales. Despite proposed correlations with traditional geotechnical parameters (see Romero et al. 2011; Della Vecchia et al. 2015), when numerical analyses at the large scale are of concern, a heuristic approach in modelling the suction and the degree of saturation calibrated on the specific problem, hence disregarding the evolution of the water retention properties with the hydromechanical state of the barrier (see Dupray et al. 2011; Gens et al. 2011; Sánchez et al. 2012; Thomas et al. 2013, among others). 
In this paper, experimental observations on the water retention behaviour of compacted bentonites are firstly presented and interpreted in the light of the material microstructure evolution. The essential features are highlighted and used for the development of a new water retention model dedicated to compacted bentonite-based materials. The aim of the novel model is twofold. On the one hand, it aims at interpreting all experimental data within a unified framework, and predicting the water retention behaviour of compacted bentonites by considering the proper physical processes in each structural level, namely adsorption in the intra-aggregate pore space and capillarity in the inter-aggregate one. On the other hand, the model provides a simple tool, characterized by a limited number of parameters, suitable for its implementation in numerical codes aimed at performing real scale simulations. Remarkably, some of the model parameters introduced have been shown to take approximately the same value for several bentonites, providing a significant basis for preliminary design when dedicated experiments are missing.

\section{Experimental observations on the retention properties of compacted bentonites}

\section{Water retention domains}

The existence of two water retention domains in compacted bentonite-based materials has been highlighted by several Authors (see for instance Lloret et al. 2003; Villar 2007; Loiseau et al. 2002; Agus et al. 2013; Seiphoori et al. 2014; Wan et al. 2015; Gatabin et al. 2016). Experimental data show that:

- For high suction values, the amount of water stored in the soil is not affected by the dry density. In this domain, water is mainly adsorbed at the surface of the clay particles and water retention is mainly controlled by the physicochemical properties of the clay minerals, in particular the specific surface (Tuller and Or 2005). This 
retention domain of intra-aggregate governing suction is often referred to as the microstructural water retention domain.

For lower suction values, the water content is sensitive to variations in dry density. Water is believed to be stored by capillarity in the macropores whose volume is affected by changes in dry density. The suction range in which the water content is affected by the dry density is called the macrostructural water retention domain.

An example is provided in Figure 1 for Febex bentonite (Lloret et al. 2003). Samples of Febex bentonite were compacted to different dry densities and then wetted under confined or unconfined conditions. Figure 1 shows that, up to a suction of about $15 \mathrm{MPa}$, the water retention curve is independent from the dry density, while for lower values of suction, it becomes dry density dependent.

Microstructural analyses performed by Lloret and Villar (2007) on compacted Febex bentonite highlighted the presence of at least two structural levels in the material. In particular, mercury intrusion porosimetry and electron microscopy data evidenced the presence of porous clay aggregates separated by inter-aggregate pores. Lloret and Villar (2007) showed that the microstructural features were independent from the compaction effort, while the volume and size of inter-aggregate pores decreased for increasing compaction effort.

\section{Effect of initial dry density}

The effect of dry density on the bentonite structure is essentially related to changes in the inter-aggregate pore volume and the pore size distribution in this domain. Within a relatively large range of dry densities and water contents, it has been observed that the compaction process mainly affects the macrostructural pore volume (Lloret et al. 2003;

Lloret and Villar 2007; Wang et al. 2013; Saba et al. 2014). Therefore, changes in dry density mainly affect the inter-aggregate water retention domain where water is stored by 
capillarity. When the density increases, not only the total volume of the voids is reduced, but also the size of the macro-pores decreases. Accordingly, pores can sustain a higher suction before emptying. From a phenomenological point view, a reduction in dry density implies a reduction of the air-entry (respectively air-occlusion) value of the material defined, along a drying (respectively wetting) path, as the suction beyond which air breaks into the saturated porous space (Della Vecchia et al. 2015).

The influence of the dry density on the water retention curve of compacted MX-80 bentonite has been recently studied by Seiphoori et al. (2014). Figure 2 presents the water retention curves, obtained for three different values of dry density, in terms of both water content and degree of saturation evolution with suction. When the water content is used to represent the quantity of water in pores (Figure 2(a)), it is evident that the larger the void ratio, the greater the quantity of water that can be stored in the material in quasi-saturated conditions. On the other hand, when the results are displayed in terms of degree of saturation (Figure 2(b)), the water retention curves of samples compacted at different dry densities are normalized in terms of pore volume and the effect of the dry density on the air-occlusion value is easier to observe.

In order to further highlight this influence, Figure 3 presents the evolution of the airocclusion value $s_{A O}$ as a function of the void ratio $e$. The data can simply be fitted by using a power law of the form:

$$
s_{A O}=\left(\frac{A}{e}\right)^{B}
$$

where $A$ and $B$ are material parameters. Such a law has been adopted for instance by Gallipoli et al. (2003), Tarantino and De Col (2008) and Gallipoli (2012) in water retention models and it is consistent with the proposals of Romero et al. (2011) and Della Vecchia et al. (2015). The nature of equation (1) is basically phenomenological and related to the hydraulic response of the porous medium at the laboratory scale. However, it implicitly describes the role of macrostructure on the hydraulic properties. 
Finally, another peculiar effect related to the role of dry density on the retention response of compacted active clays have been highlighted by Cui et al. (2002). The Authors performed a wetting-drying suction-controlled cycle on a sample of FoCa7 clay compacted at a high dry density $\left(\rho_{d}=1.85 \mathrm{Mg} / \mathrm{m}^{3}\right)$ and monitored the evolution of both void ratio and water content. During the test, the sample was left free to swell (zero applied stress). Test results show reversibility in terms of water content and void ratio evolution against with suction, which can be interpreted as the macroscopic consequence of the predominance of physico-chemical effects. Physico-chemical effects, like water adsorption onto clay minerals governing the intra-aggregate retention region, are in fact mainly reversible, while capillary retention mechanism are known to be related to the irreversibility of the water retention response of granular material. For heavily compacted material, the inter-aggregate pore space can be considered negligible, as well as the water stored in the inter-aggregate retention region. As a consequence, both the mechanical and hydraulic response is governed by the reversible aggregate swelling-shrinking behaviour. The same reversibility is found by the Authors also if suction changes are performed at different vertical stresses, namely 10 and $20 \mathrm{MPa}$. Reversibility in terms of retention response is lost if capillary mechanisms are present, i.e. if similar tests are performed on looser swelling clays (as in Chu and Mou 1973; Alonso et al. 1995).

\section{Effect of the volume constraints}

A specific feature of compacted bentonite-based materials is the important sensitivity of their water retention properties to the imposed volume constraints. Experimental data on compacted bentonites show that, for a given suction, the quantity of water stored under free swelling conditions is greater than it is under prevented swelling (Loiseau et al. 2002; Lloret et al. 2003; Ye et al. 2009; Wang et al. 2013; Seiphoori et al. 2014; Gatabin et al. 2016). In bentonites, coupled hydromechanical processes are indeed extremely strong, so 
that under free swelling conditions, suction changes yield significant swelling or shrinkage (in the order of several tens, even hundreds percent of the initial volume), resulting in important changes of dry density.

185 effects of volume constraints on the water retention behaviour of compacted bentonites. Let us write the degree of saturation as the ratio between the water ratio $e_{w}$ (volume of water over solid volume), and the void ratio $e$

$$
S_{r}=\frac{e_{w}}{e}
$$

Equation (2) evidences the dependence of the degree of saturation on both water ratio 190

and void ratio. In particular, Tarantino (2009) introduced the concept of hydraulic wetting to describe an increase of the degree of saturation due to an increase of the water ratio. Accordingly, the term mechanical wetting was used for an increase of the degree of saturation resulting from a decrease of the void ratio.

Competing effects of volume change and water uptake on the water retention behaviour were highlighted by Gatabin et al. (2016). The Authors investigated the water retention properties of a compacted mixture of MX-80 bentonite/sand mixture (with a respective proportion of 70/30 in dry mass) under both free swelling and constant volume conditions. As can be observed in Figure 4(a), the imposed volume constraint significantly impacts the water retention behaviour of the mixture compacted at a dry density of $2.03 \mathrm{Mg} / \mathrm{m}^{3}$. For the samples wetted under confined conditions, hydration led to an increase of the degree of saturation. In this case, only hydraulic wetting takes place and the degree of saturation univocally increases. On the contrary, the decrease of suction did not significantly impact the degree of saturation of the samples wetted under free conditions. During hydration under free swelling conditions, swelling strains indeed develop as the material takes water, 
and both water ratio and void ratio are affected (Figure 4(b). In this case, hydraulic wetting occurs simultaneously as mechanical drying.

\section{Model formulation}

Based on the experimental observations and the water retention mechanisms thoroughly described in the previous section, a water retention model for compacted bentonites is developed. The model is developed in order to consider explicitly the physical processes that characterize the two structural levels of compacted bentonites: adsorption in the intra-aggregate pore space and capillarity in the inter-aggregate one. Introducing such a distinction between the retention mechanisms inside and between the aggregates allows for the reproduction of the density dependence of the retention behaviour when a storage mechanism dominates, i.e. for low suctions, while the degree of saturation of the micropores is solely a function of the suction. As a consequence, loading path dependency, e.g. the different hydraulic response of samples saturated with different mechanical constraints, and the transition in retention properties from low to high density prepared sample are naturally embedded in the model. The necessity of simulating different physical processes in the two domains is just a seeming complication of the existing frameworks, performed to the aim of keeping the number of model parameters as limited as possible, as well as to find some parameters which can be considered, as a first approximation, constant for any bentonite.

Accordingly, the proposed water retention model is formulated in terms of water ratio $e_{w}$ in order to evidence the role of the different water retention mechanisms, namely adsorption in the microstructure (inter-layer porosity and inter-particle porosity) and capillary storage in the inter-aggregate porosity, according to the framework proposed in Romero and Vaunat (2000) and Romero et al. (2011). The water ratio $e_{w}$ is thus expressed as the sum of a contribution from the water stored in the micropores $e_{w m}$ and a second 
contribution from the water contained in the macropores $e_{w M}$ (Figure 5)

$$
e_{w}=e_{w m}+e_{w M}
$$

It should be mentioned that the volume of adsorbed water content may be greater than the intra-aggregate pore volume as measured in mercury intrusion porosimetry tests. Indeed some water is adsorbed at the surface of the aggregates (Lloret et al. 2003). Yet this phenomenon is not explicitly taken into account in the present model.

The degree of saturation $S_{r}$ is then expressed as

$$
S_{r}=\frac{e_{w}}{e}=\frac{e_{m}}{e} S_{r m}+\frac{e_{M}}{e} S_{r M}
$$

where $S_{r m}$ and $S_{r M}$ are respectively the microstructural and macrostructural degrees of saturation. $S_{r m}$ is defined as the ratio between the volume of water in the intra-aggregate pores and the volume of the intra-aggregate pores themselves, while $S_{r M}$ is defined as the volume of water in the inter-aggregate pores divided by the volume of the inter-aggregate pores. The degrees of saturation are therefore not additive, as the global degree of saturation is obtained by the sum of the microstructural and macrostructural degrees of saturation, weighted by the corresponding volumetric fractions. The microstructural and macrostructural degrees of saturation are not primary variables of the retention model, which is defined in terms of water ratios, but they can be calculated a posteriori once the water ratios $\left(e_{w m}\right.$ and $\left.e_{w M}\right)$ and the volume of the intra- and inter-aggregate pore space $\left(e_{m}\right.$ and $\left.e_{M}=e-e_{m}\right)$ are known.

In the following, thermodynamic equilibrium between the microstructure and macrostructure is assumed. Accordingly, the current value of suction applies to both structural levels. The formulation of the proposed water retention model includes three parts, namely the descriptions of the microstructural water retention domain, the macrostructural domain 
and the microstructure evolution.

\section{Microstructural water retention domain}

Water in the microstructure is mainly stored by adsorption. Several adsorption isotherms have been proposed in the literature by the community of physicists. In particular, Dubinin's theory (Dubinin and Radushkevich 1947) was developed for activated carbon and zeolites, which contain cavities of molecular dimensions. The micropores of these materials control their adsorptive nature. Later, Kraehenbuehl et al. (1987) used Dubinin's theory to describe the bentonite - water system and Fernández and Rivas (2005) to model the water retention behaviour of Febex bentonite.

In this paper, Dubinin's isotherm is adopted to describe the water retention behaviour of the microstructure. Its equation takes the form

$$
\Omega_{w m}=\Omega_{m} \exp \left\{-\left[\frac{R T}{\beta_{D} E_{0}} \ln \left(\frac{u_{v}^{0}}{u_{v}}\right)\right]^{n_{a d s}}\right\}
$$

where $\Omega_{w m}$ is the volume of water adsorbed in the micropores at temperature $T$ and relative pressure $u_{v} / u_{v}^{0}, R$ is the universal gas constant $(=8.314 \mathrm{~J} / \mathrm{mol} \cdot \mathrm{K})$, and $\Omega_{m}$ is the total volume of the micropores, $n_{a d s}$ is a specific parameter of the system, called heterogeneity factor. $\beta_{D}$ is termed similarity constant and $E=\beta_{D} E_{0}$ is the characteristic adsorption energy for the given system. $E_{0}$ is the characteristic energy of adsorption for a reference vapour for which $\beta_{D}=1$.

Equation (5) may be expressed in terms of water ratio by dividing both sides of the 275 equation by the the volume of solid particles $\Omega_{s}$. It yields

$$
e_{w m}=e_{m} \exp \left\{-\left[\frac{R T}{\beta_{D} E_{0}} \ln \left(\frac{u_{v}^{0}}{u_{v}}\right)\right]^{n_{a d s}}\right\} \text {. }
$$

Furthermore, Kelvin's law may be used to express the relative pressure $u_{v} / u_{v}^{0}$ in terms of 
suction $s$

$$
R H=\frac{u_{v}}{u_{v}^{0}}=\exp \left(\frac{-s M_{w}}{R T \rho_{w}}\right)
$$

where $R H$ is the relative humidity, $M_{w}$ is the molecular mass of water $(=0.018 \mathrm{~kg} / \mathrm{mol})$ and $\rho_{w}$ is the water density. Gathering the constant parameters, the following expression 280 is finally adopted for the microstructural water retention domain

$$
e_{w m}\left(s, e_{m}\right)=e_{m} \exp \left[-\left(C_{a d s} s\right)^{n_{a d s}}\right]
$$

where $n_{a d s}$ and $C_{a d s}$ are material parameters. The parameter $n_{a d s}$ controls the curvature of the water retention curve in the high suction range, while $C_{a d s}$ is associated to the air-entry (or air-occlusion) suction of the intra-aggregate voids (Figure 6). It is related to the original Dubinin equation through

$$
C_{a d s}=\frac{M_{w}}{\rho_{w} \beta_{D} E_{0}} .
$$

For typical values of $E=\beta_{D} E_{0}$ ranging between 1 and $10 \mathrm{~kJ} / \mathrm{mol}, C_{a d s}$ varies between 0.018 and $0.0018 \mathrm{MPa}^{-1}$. According to our proposal, the microstructural water content is assumed to depend just on the microstructural void ratio (i.e. on the size of the aggregate) and on suction. Direct dependence on soil dry density is thus disregarded.

\section{Macrostructural water retention domain}

290 The van Genuchten (1980) water retention model has been successfully used to model the water retention behaviour of a wide variety of soils. It is generally expressed as

$$
S_{r}(s)=\left[1+\left(\frac{s}{\alpha}\right)^{n}\right]^{-m}
$$


where $m$ and $n$ are material parameters, and $\alpha$ is related to the air-entry value. Alternatively, the van Genuchten equation may expressed in terms of water ratio $e_{w}$

$$
e_{w}(s, e)=e\left[1+\left(\frac{s}{\alpha}\right)^{n}\right]^{-m}
$$

where $e$ is the void ratio.

295

In this paper, the van Genuchten equation is selected to model the macrostructural water retention domain. Accordingly, the void ratio $e$ is replaced by the macrostructural void ratio $e_{M}=e-e_{m}$, and the macrostructural water retention model reads

$$
e_{w M}\left(s, e, e_{m}\right)=\left(e-e_{m}\right)\left[1+\left(\frac{s}{\alpha}\right)^{n}\right]^{-m}
$$

In order to represent the influence of the bentonite structure on the air-entry value, the parameter $\alpha$ is assumed to depend on the macrostructural void ratio.

Following a purely phenomenological approach, although experimentally validated in Della Vecchia et al. (2015) and Dieudonne et al. (2014b) by means of MIP data, the air-entry value is supposed to vary with the inter-aggregate void ratio according to the 305 relation

$$
\alpha=\frac{A}{e-e_{m}}
$$

where $A$ controls the dependence of the air-entry pressure on the macrostructural void ratio. This relationship is similar to Equation (1) for $B=1$, while accounting for the double structure of compacted bentonite. Accordingly, the influence of the void ratio on the macrostructural water retention curve is clearly related to the air-entry (or air310 occlusion) value of the material: the lower the dry density, the lower the suction needed to empty the macro-pores. 


\section{Microstructure evolution}

The microstructure of bentonite is significantly affected by changes of its water content. In particular, the evolution of the microstructure during wetting or drying paths may be

\section{Calibration procedure}

335 It reads macrostructural void ratio.

characterized by the evolution of the microstructural void ratio $e_{m}$. In order to account for structural changes of the material along the water retention curve, the microstructural model proposed in Della Vecchia et al. (2015) is introduced in the water retention model.

$$
e_{m}=\beta_{0} e_{w}^{2}+\beta_{1} e_{w}+e_{m 0}
$$

where $e_{m 0}$ is the microstructural void ratio for the dry material $\left(e_{w}=0\right)$ and $\beta_{0}$ and $\beta_{1}$ are parameters that quantify the swelling potential of the aggregates. These parameters are determined for Febex bentonite using experimental data from Lloret et al. (2003), Lloret and Villar (2007) and Romero et al. (2011), for MX-80 bentonite using experimental data from Delage et al. (2006), Wang (2012) and Seiphoori et al. (2014), and for a mixture of MX-80 bentonite and sand using data from Wang et al. (2013) and Saba et al. (2014). The parameters are given in Table 1. Further details on the calibration of the microstructure evolution law (14) are given in the section entitled "Microstructure evolution parameters".

The influence of the microstructure evolution on the water retention curve is highlighted in Figure 7. In this figure, the water retention curves for two values of $e_{m}$ constant are represented, together with the water retention curve considering microstructure evolution. As observed, the increase of microstructural void ratio upon wetting under constant $e$ yields an increase of the apparent air-entry value as a consequence of the decrease of the 
- $C_{a d s}$ and $n_{a d s}$ to describe the water retention response of the intra-aggregate pores,

- $A, n$ and $m$ to describe the water retention response of the inter-aggregate retention region,

- $\beta_{0}, \beta_{1}$ and $e_{m 0}$ to characterize the evolution of the microstructural void ratio with the water ratio.

\section{Microstructure evolution parameters}

The parameters of the evolution law of intra-aggregate void ratio with water content (Equation (14)) can be estimated first, independently from the other parameters. They can be directly estimated just if experimental pore size distributions (PSD) are available for the material at different water content, by interpreting the experimental PSD curves in the framework of double porosity media, i.e. making a distinction between micropores and macropores. From each pore size distribution (corresponding to a given water content of the material) a value of $e_{m}$ can be estimated, obtaining one point in the $\left(e_{w}-e_{m}\right)$ plane (Della Vecchia et al. 2015). The parameters of the evolution law can then be determined by best fitting. Details on distinction criteria between intra- and inter-aggregate pores from MIP results can be found in Romero et al. (2011).

An alternative way of obtaining a first estimate of the parameters of Equation (14) comes from the correlations between these parameters and soil properties (like specific surface or activity index), as presented in Romero et al. (2011) and Della Vecchia et al. (2015).

\section{Microstructural water retention parameters}

The microstructural parameters $C_{a d s}$ and $n_{a d s}$ control the water retention behaviour at high values of suction. The calibration of these parameters is made easier by presenting experimental data in the $\left(s-e_{w}\right)$ plane, where the independence on dry density is high-

lighted. At high suction values, a collection of points in this plane (also corresponding to 
different dry densities) is sufficient to calibrate the two parameters. In particular, Figure 8 shows how the parameter $C_{a d s}$ controls the slope of the water retention curve in the high suction range: the higher $C_{a d s}$, the steeper the slope of the water retention curve in the $\left(s-e_{w}\right)$ plane. Finally, the value of $e_{m}$ required to evaluate $e_{w m}$ can be considered known for a given water content once that the calibration described in the previous section has been performed.

\section{Macrostructural water retention parameters}

In order to calibrate the macrostructural water retention model, experimental data for different dry densities are required. The macroscopic parameter $A$ allows for tracking the dependency of the water retention on void ratio: it can reproduce the correct evolution of the air-entry (or air-occlusion) value of the material with dry density. The parameters $n$ and $m$ influence the desiccation/imbibition rate of the material in the low suction range. Remarkably, they have shown to hardly vary from one material to another (see the next section). The following values can be used in a first approximation: $n=3$ and $m=0.15$.

\section{${ }_{375}$ Experimental validation}

The proposed water retention model is validated against experimental data from the literature. Attention is focused on bentonite-based materials in which coupled hydromechanical phenomena are extremely strong. Yet the model can be used for compacted clays with lower activity and which display an aggregated structure upon compaction.

\section{Febex bentonite}

Villar (2000) and Lloret et al. (2005) determined the water retention curves of Febex bentonite compacted to different dry densities, namely $1.60,1.65$ and $1.70 \mathrm{Mg} / \mathrm{m}^{3}$. Compacted samples were hydrated under both confined and unconfined conditions. Under unconfined conditions, the dimensions of the samples were measured in order to deter- 
mine their density, hence their degree of saturation.

The water retention curves under confined conditions (i.e. at constant dry density) are used to calibrate the water retention model. The calibrated parameters are: $C_{a d s}=0.0028$ $\mathrm{MPa}^{-1}, n_{a d s}=0.78, A=0.24 \mathrm{MPa}, n=3$ and $m=0.15$. As shown in Figure 9, the model succeeds in capturing the increase of air-occlusion pressure with increasing dry density.

The model is then validated against data under unconfined conditions (Figure 10(a)). In this figure, experimental data are reported as empty symbols, while model predictions are plotted as black symbols. Different shapes of the symbols correspond to different dry densities.

In this case, the values of void ratio determined experimentally were used to compute the degree of saturation. A fairly good agreement is obtained for samples wetted under free volume conditions, although overestimation of the degree of retention becomes significant for suctions below $2 \mathrm{MPa}$. Several reasons can explain this discrepancy. First of all, the evolution of the microstructural void ratio in the range of high water contents is not well characterized, so that uncertainties on the microstructure evolution are high in this domain. Secondly, the low suction range corresponds to the domain where the volume changes are the most important and where the uncertainties on the measurements are the largest. Figure 10(b) presents the evolution of the void ratio upon wetting. As can be observed in the figure, hydration yields important volume changes, with the void ratio reaching a value of 1.6. This very large increase of the porous volume significantly affects the water retention behaviour of Febex bentonite. For the sake of illustration, the water retention curves predicted by the model considering two constant dry densities (the initial and final ones) are represented in Figure 10(a) by the dotted and continuous lines, respectively. Comparison between these curves and the experimental data proves that a model 
that cannot account for dry density variations would provide unsatisfactory prediction, both from the qualitative and the quantitative point of view: an increase in the void ratio 415 yield a decrease of the air-entry value and modifies the slope of the curve in the $\left(s-S_{r}\right)$ plane.

Finally, the model is used to reproduce the water retention behaviour of granular Febex bentonite (Figure 11(a). This material, investigated by Alonso et al. (2011), consists in a mixture of bentonite pellets of very high dry densities (up to $1.95 \mathrm{Mg} / \mathrm{m}^{3}$ ). Accordingly, three pore families may be distinguished, namely micropores and macropores in the bentonite pellets, and large pores between the pellets.

The water retention curves for two dry densities of the mixture were determined under constant volume conditions. Figure 11(b) compares the experimental data with the model predictions. It is worth noting that the parameters used for this simulation are the ones calibrated on Villar (2000) and Lloret et al. (2005) data and no dedicated calibration has been performed. Although developed within a double-porosity framework, the model succeeds in tracking the evolution of the degree of saturation upon wetting under constant volume conditions. In the high suction range, the water retention behaviour of the mixture is mainly controlled by the bentonite pellets. On the other hand, the large inter-pellet pores do not significantly affects the water retention behaviour of the material as they tend to disappear upon isochoric wetting.

\section{MX-80 bentonite}

Villar (2004) investigated the water retention properties of MX-80 bentonite under confined conditions. Samples of MX-80 bentonite were uniaxially compacted to different dry densities and water contents, and suction was then measured.

Figure 12 represents the experimental data in the $\left(s-S_{r}\right)$ plane together with the model 
440

predictions. The model is calibrated using experimental data for $\rho_{d}=1.50 \mathrm{Mg} / \mathrm{m}^{3}$ and $\rho_{d}=1.80 \mathrm{Mg} / \mathrm{m}^{3}$, and validated against data for $\rho_{d}=1.60 \mathrm{Mg} / \mathrm{m}^{3}$ and $\rho_{d}=1.70 \mathrm{Mg} / \mathrm{m}^{3}$. The calibrated parameters are: $C_{a d s}=0.0075 \mathrm{MPa}^{-1}, n_{a d s}=1.5, A=0.2 \mathrm{MPa}, n=3$ and $m=0.15$. As observed in Figure 12, the degrees of saturation estimated by the water retention model compare favourably with the measured degrees of saturation. In addition, the evolution of the air-occlusion value is consistent with the data obtained by Seiphoori et al. (2014).

\section{MX-80 bentonite/sand mixture}

The water retention properties of a mixture of $70 \%$ MX- 80 bentonite and $30 \%$ sand mixture were studied by Gatabin et al. (2016) and Wang et al. (2013) under both confined and unconfined conditions. Figure 13 presents the experimental water retention curves obtained under confined conditions, together with the model calibration. The calibrated parameters are: $C_{a d s}=0.0053 \mathrm{MPa}^{-1}, n_{a d s}=0.79, A=0.2 \mathrm{MPa}, n=3$ and $m=0.15$. The model provides excellent fitting of the experimental data.

The model is validated against experimental data on wetting paths under unconfined conditions. Figures 14(a) and 14(b) present the evolution of the degree of saturation of the compacted mixtures upon wetting. In both cases, the model remarkably succeeds in tracking the evolution of the degree of saturation over the whole range of investigated suctions. Note that, in Figure 14(b), experimental data and model predictions are superposed at suctions of $150 \mathrm{MPa}, 71 \mathrm{MPa}, 48 \mathrm{MPa}, 38 \mathrm{MPa}$ and $13 \mathrm{MPa}$. For the sake of completeness, the water retention curves predicted for the initial and final dry densities (taken as constant during the simulation) are also represented in Figure 14(a), and for the different current void ratios in Figure 14(b). As observed in Figure 14(b), an important decrease in the air-entry pressure is associated with the important swelling of the dense material. 
In order to better understand the mechanisms behind the competing effects, the evolution of the total, microstructural and macrostructural void ratios upon wetting is analysed. Under confined conditions, wetting leads to an increase of the microstructural void ratio 470 (following the evolution predicted by Equation (14)), hence a decrease of the macroporous volume (Figure 15(a)). On the other hand, when the sample is wetted under free swelling conditions, the overall swelling of the sample is more important than the development of the microstructure (Figure 15(b)). An increase in the inter-aggregate volume is predicted.

\section{Summary}

Table 2 presents the values of the model parameters calibrated for three bentonite-based materials, namely Febex and MX-80 bentonites, and a mixture of MX-80 bentonite and sand. As can be observed, the macrostructural parameters hardly depend on the considered material and the values of $A=0.2 \mathrm{MPa}, n=3$ and $m=0.15$ may be assumed as a first approximation. A possible explanation for this observation is that the macrostructural parameters are rather affected by the compaction process than the physicochemical properties of the material. On the other hand, the microstructural parameters vary for the different materials. It is likely that they depend on physicochemical properties of the materials, such as the specific surface area and the cation exchange capacity.

\section{Conclusions}

In this paper, the main features of the water retention properties of compacted bentonites are first reviewed and explained at the light of the bentonite structure. Owing for strong multi-physical and multi-scale coupled processes, a particularity of bentonites is that the density of the material is evolving not only along mechanical paths, but foremost upon wetting and drying.

Based on experimental observations at both micro and macro scales, a phenomenolog- 
ical water retention model is developed. The model accounts for the double structure of compacted bentonite-based materials, and its evolution along hydromechanical stress paths. Proper water retention processes in each structural level are considered, namely adsorption in the intra-aggregate pore space and capillarity in the inter-aggregate one.

The model succeeds in representing the water retention behaviour of bentonite-based materials compacted to different dry densities and wetted under both confined and unconfined conditions. The model provides a better understanding of the influence of the complex hydromechanical processes on the water retention curve, interpreting all experimental data within a unified framework. In addition, its simplicity, together with the limited number of parameters, make the model suitable for its implementation in numerical codes aimed at performing real scale simulations. Remarkably, some of the model parameters introduced have indeed been shown to take approximately the same value for several bentonites, providing a significant basis for preliminary design when dedicated experiments are missing.

\section{Acknowledgements}

The Authors wish to acknowledge the fruitful discussions with Prof. Enrique Romero from Technical University of Catalonia (Spain). The first author is grateful for financial support 510 from the FRIA-F.R.S.-FNRS, the National Fund of Scientific Research in Belgium. 


\section{References}

Agus, S. S., Arifin, Y. F., Tripathy, S. and Schanz, T. 2013. Swelling pressure - suction relationship of heavily compacted bentonite-sand mixtures. Acta Geotechnica 8, No. 2, $155-165$.

515

Alonso, E. E., Lloret, A., Gens, A. and Yang, D. Q. 1995. Experimental behaviour of highly expansive double-structure clay. In Proceedings of the First International Conference on Unsaturated Soils, UNSAT 1995 (Alonso, E. E. and Delage, P., eds.), Paris, France, pp. 11-16.

Alonso, E. E., Romero, E. and Hoffmann, C. 2011. Hydromechanical behaviour of compacted granular expansive mixtures: experimental and constitutive study. Géotechnique 61, No. 4, 329-344.

Arroyo, H., Rojas, A., de la Luz Pérez-Rea, M. and Arroyo, J. 2015. A porous model to simulate the evolution of the soil-water characteristic curve with volumetric strains. Comptes Rendus Mécanique 343, No. 4, 264-274.

Beckett, C. T. S. and Augarde, C. E. 2013. Prediction of soil water retention properties using pore-size distribution and porosity. Canadian Geotechnical Journal 50, No. 4, 435-450.

Brooks, R. N. and Corey, A. T. 1964. Hydraulic properties of porous media. Technical report, Colorado State University Hydrology Paper No. 3.

Casini, F., Vaunat, J., Romero, E. and Desideri, A. 2012. Consequences on water retention properties of double-porosity features in a compacted silt. Acta Geotechnica 7, 139-150.

Chu, T. Y. and Mou, C. H. 1973. Volume change characteristics of expansive soils determined by controlled suction. In Proceedings of the Third International Conference on Expansive Soils, Haifa, Israel, pp. 177-185. 
Cui, Y. J., Yahia-Aissa, M. and Delage, P. 2002. A model for the volume change behavior of heavily compacted swelling clays. Engineering Geology 64, No. 2-3, 233-250.

Delage, P., Marcial, D., Cui, Y. J. and Ruiz, X. 2006. Ageing effects in a compacted bentonite: a microstructure approach. Géotechnique 56, No. 5, 291-304.

Della Vecchia, G., Dieudonne, A. C., Jommi, C. and Charlier, R. 2015. Accounting for evolving pore size distribution in water retention models for compacted clays. International Journal for Numerical and Analytical Methods in Geomechanics 39, No. 7, $702-723$.

Della Vecchia, G., Jommi, C. and Romero, E. 2013. A fully coupled elastic-plastic hydromechanical model for compacted soils accounting for clay activity. International Journal for Numerical and Analytical Methods in Geomechanics 37, No. 5, 503-535.

Dieudonne, A., Charlier, R., Levasseur, S., Della Vecchia, G. and Jommi, C. 2014a. Evolution of clay fabric and water retention properties along hydromechanical stress paths. In Numerical Methods in Geotechnical Engineering, Proceedings of the 8th European Conference on Numerical Methods in Geotechnical Engineering, NUMGE 2014, Delft, The Netherlands, pp. 971-975.

Dieudonne, A., Della Vecchia, G., Charlier, R. and Jommi, C. 2014b. Influence of microfabric evolution on the retention behaviour of compacted clayey soils. In Unsaturated Soils: Research and Applications - Proceedings of the 6th International Conference on Unsaturated Soils, UNSAT 2014, Sydney, Australia, pp. 679-684.

5 Dubinin, M. M. and Radushkevich, L. V. 1947. Equation of the characteristic curve of activated charcoal. Proceedings of the Academy of Sciences, Physical Chemistry Section, USSR 55, 331-333.

Dupray, F., François, B. and Laloui, L. 2011. Analysis of the FEBEX multi-barrier system including thermoplasticity of unsaturated bentonite. International Journal for Numerical and Analytical Methods in Geomechanics 37, No. 4, 399-422. 
Fernández, A. M. and Rivas, P. 2005. Analysis and distribution of waters in the compacted FEBEX bentonite: pore water chemistry and adsorbed water properties. In Proceedings of International Symposium on large scale field tests in granite: Advances in understanding engineered clay barriers (Alonso, E. E. and Ledesma, A., eds.), Sitges, Spain, pp. 257-276.

Fredlund, D. G. and Xing, A. 1994. Equations for the soil-water characteristic curve. Canadian Geotechnical Journal 31, No. 3, 521-532.

Gallipoli, D. 2012. A hysteretic soil-water retention model accounting for cyclic variations of suction and void ratio. Géotechnique 62, No. 7, 605-616.

Gallipoli, D., Wheeler, S. J. and Karstunen, M. 2003. Modelling the variation of degree of saturation in a deformable unsaturated soil. Géotechnique 53, No. 1, 105-112.

Gardner, W. R. 1958. Some steady-state solutions of the unsaturated moisture flow equation with application to evaporation from a water table. Soil Science $\mathbf{8 5}$, No. 4, 228-232.

Gatabin, C., Talandier, J., Collin, F., Charlier, R. and Dieudonné, A. C. 2016. Competing effects of volume change and water uptake on the water retention behaviour of a compacted mx-80 bentonite/sand mixture. Applied Clay Science 121-122, 57-62.

Gens, A., Vallejan, B., Sánchez, M., Imbert, C., Villar, M. V. and Van Geet, M. 2011. Hydromechanical behaviour of a heterogeneous compacted soil: experimental observations and modelling. Géotechnique 61, No. 5, 367-386.

He, Y., G., C. Y., Ye, W. M., Chen, B. and Cui, Y. C. 2016. Influence of salt concentration on volume shrinkage and water retention characteristics of compacted gmz bentonite. Environmental Earth Sciences 75, No. 6, 1-10.

Hu, R., Chen, Y. F., Liu, H. H. and Zhou, C. B. 2013. A water retention curve and unsaturated hydraulic conductivity model for deformable soils: consideration of the change in pore-size distribution. Géotechnique 63, No. 16, 1389-1405. 
Kraehenbuehl, F., Stoeckli, H. F., Brunner, F., Kahr, G. and Mueller-Vonmoos, M. 1987. Study of the water - bentonite system by vapour adsorption, immersion calorimetry and X-ray techniques: I. Micropore volumes and internal surface areas, following Dubinin's theory. Clay Minerals 22, No. 1, 1-9.

Lloret, A., Romero, E. and Villar, M. V. 2005. FEBEX II Project. Final report on thermohydro-mechanical laboratory tests. Technical report, ENRESA.

Lloret, A. and Villar, M. V. 2007. Advances on the knowledge of the thermo-hydromechanical behaviour of heavily compacted FEBEX bentonite. Physics and Chemistry of the Earth 32, No. 8-14, 701-715.

Lloret, A., Villar, M. V., Sánchez, M., Gens, A., Pintado, X. and Alonso, E. E. 2003. Mechanical behaviour of heavily compacted bentonite under high suction changes. Géotechnique 53, No. 1, 27-40.

Loiseau, C., Cui, Y. J. and Delage, P. 2002. The gradient effect on the water flow through a compacted swelling soil. In Proceedings of the Third International Conference on Unsaturated Soils, UNSAT 2002 (Juca, J., de Campos, T. and Marinho, F., eds.), Recife, Brazil, pp. 395-400.

Masin, D. 2010. Predicting the dependency of a degree of saturation on void ratio and suction using effective stress principle for unsaturated soils. International Journal for Numerical and Analytical Methods in Geomechanics 34, 73-90. in bentonite-sand mixtures. In Proceedings of the Third International Conference on Unsaturated Soils, UNSAT 2002 (Juca, J. F. T., de Campos, T. M. P. and Marinho, F. A. M., eds.), Recife, Brazil, pp. 283-288.

Mbonimpa, M., Aubertin, M., Maqsoud, A. and Buissière, B. 2006. Predictive model for the water retention curve of deformable clayey soils. Journal of Geotechnical and Geoenvironmental Engineering 132, No. 9, 1121-1132. 
Nuth, M. and Laloui, L. 2008. Effective stress concept in unsaturated soils: clarification and validation of a unified framework. International Journal for Numerical and Analytical Methods in Geomechanics 32, No. 7, 771-801.

615

620

Romero, E., Della Vecchia, G. and Jommi, C. 2011. An insight into the water retention properties of compacted clayey soils. Géotechnique 61, No. 4, 313-328.

Romero, E. and Vaunat, J. 2000. Retention curves of deformable clays. In Experimental Evidences and Theoretical Approaches in Unsaturated Soils (Tarantino, A. and Mancuso, C., eds.), Trento, Italy, pp. 91-106.

Saba, S., Delage, P., Lenoir, N., Cui, Y. J., Tang, A. M. and Barnichon, J. D. 2014. Further insight into the microstructure of compacted bentonite-sand mixture. Engineering Geology 168, 141-148.

Seiphoori, A., Ferrari, A. and Laloui, L. 2014. Water retention behaviour and microstructural evolution of MX-80 bentonite during wetting and drying cycles. Géotechnique 64, No. $9,721-734$.

Simms, P. H. and Yanful, E. K. 2002. Predicting soil-water retention characteristic curves of compacted plastic soils from measured pore-size distributions. Géotechnique 52, No. 4, 269-278.

Sánchez, M., Gens, A. and Olivella, S. 2012. THM analysis of a large-scale heating test incorporating material fabric changes. International Journal for Numerical and Analytical Methods in Geomechanics 36, No. 4, 391-421.

Tarantino, A. 2009. A water retention model for deformable soils. Géotechnique 59, No. 9, $751-762$.

Tarantino, A. and De Col, E. 2008. Compaction behaviour of clay. Géotechnique 58, No. 3, 199-213. 
Thom, R., Sivakumar, R., Sivakumar, V., Murray, E. J. and Mackinnon, P. 2007. Pore size distribution of unsaturated compacted kaolin: the initial states and final states following saturation. Géotechnique 57, No. 5, 469-474.

Thomas, H. R., Vardon, P. J. and Cleall, P. J. 2013. Three-dimensional behaviour of a pro640 totype radioactive waste repository in fractured granitic rock. Canadian Geotechnical Journal 51, No. 3, 246-259.

Thyagaraj, T. and Salini, U. 2016. Effect of pore fluid osmotic suction on matric and total suctions of compacted clay. Géotechnique 65, No. 11, 952-960.

Tuller, M. and Or, D. 2005. Water films and scaling of soil characteristic curves at low water contents. Water Resources Research 41, No. W09403, 1-6.

van Genuchten, M. T. 1980. A closed-form equation for predicting the hydraulic conductivity of unsaturated soils. Soil Science Society of America Journal 44, No. 5, 892-898.

Villar, M. V. 2000. Caracterizacion thermo-hidro-mecanica de una bentonita de Cabo de Gata. Ph.D. thesis, Universidad Complutense de Madrid.

Villar, M. V. 2002. Thermo-hydro-mechanical characterisation of a bentonite from Cabo de Gata. Technical report, CIEMAT.

Villar, M. V. 2004. MX-80 bentonite. Thermo-hydro-mechanical characterization performed at CIEMAT in the context of the Prototype Project. Technical report, CIEMAT.

Villar, M. V. 2007. Water retention of two natural compacted bentonites. Clays and Clay Minerals 55, No. 3, 311-322.

Wan, M., Ye, W. M., Chen, Y. G., Cui, Y. J. and Wang, J. 2015. Influence of temperature on the water retention properties of compacted gmz01 bentonite. Environmental Earth Sciences 73, 4053-4061.

Wang, Q. 2012. Hydro-mechanical behaviour of bentonite-based materials used for high660 level radioactive waste disposal. Ph.D. thesis, Université Paris-Est. 
Wang, Q., Tang, A. M., Cui, Y. J., Delage, P., Barnichon, J. D. and Ye, W. M. 2013. The effects of technological voids on the hydro-mechanical behaviour of compacted bentonite-sand mixture. Soils and Foundations 53, No. 2, 232-245.

Ye, W. M., Cui, Y. J., Qian, L. X. and Chen, B. 2009. An experimental study of the 665 water transfer through confined compacted GMZ bentonite. Engineering Geology 108, No. 3-4, 169-176.

Zhou, A. N., Sheng, D. and Carter, J. P. 2012. Modelling the effect of initial density on soil-water characteristic curves. Géotechnique 62, No. 8, 669-680. 


\section{Figure captions}

1 Water retention curves of compacted Febex bentonite obtained under confined and unconfined conditions (modified from Lloret et al. 2003). . . . . .

2 Water retention curves of MX-80 bentonite compacted at three different dry densities (Seiphoori et al. 2014): (a) water content versus suction; (b) degree of saturation versus suction. . . . . . . . . . .

3 Evolution of the air-occlusion value of compacted MX-80 bentonite as a function of the void ratio (experimental data from Seiphoori et al. 2014).

4 Water retention behaviour of a MX-80 bentonite/sand mixture compacted at a dry density $\rho_{d}=2.03 \mathrm{Mg} / \mathrm{m}^{3}$ (Gatabin et al. 2016): (a) water retention curves obtained under confined and unconfined conditions; (b) evolution of the water and void ratios upon wetting under unconfined conditions. . . . .

5 Conceptual representation of the water retention mechanisms in compacted bentonites. . . . . . . . . . . . . . . . . .

6 Influence of the microstructural parameters on the shape of the microstructural water retention curve $\left(e_{m}=0.3\right.$ is taken constant): (a) influence of $n_{a d s}\left(C_{a d s}=0.005 \mathrm{MPa}^{-1}\right) ;(\mathrm{b})$ influence of $C_{a d s}\left(n_{a d s}=0.8\right) \ldots \ldots$. .

7 Influence of microstructure evolution on the shape of the (global) water retention curve. The parameters of the model are $C_{a d s}=0.005 \mathrm{MPa}^{-1}$, $n_{\text {ads }}=0.8, e=0.6, A=0.3 \mathrm{MPa}, n=3$ and $m=0.15 \ldots \ldots$

8 Influence of $C_{a d s}$ on the shape of the (global) water retention curve. The parameters of the model are $n_{a d s}=0.8, e=0.6, A=0.3 \mathrm{MPa}, n=3$, $m=0.15, \beta_{0}=0.25, \beta_{1}=0.05$ and $e_{m 0}=0.25 \ldots \ldots \ldots$

9 Calibration of the water retention model against experimental data (Lloret et al. 2003) on Febex bentonite compacted at three different dry densities. Wetting path under confined conditions. 
10 Comparison between experimental data (Villar 2002) and model predictions on compacted Febex bentonite. Wetting path under unconfined conditions: (a) degree of saturation; (b) void ratio (experimental). . . . . . . . .

11 Water retention properties of granular Febex bentonite (Alonso et al. 2011). The maximum pellet size is $4 \mathrm{~mm}$. (a) Photograph of the granular mixture; (b) comparison between experimental data and model predictions. . . . . .

12 Comparison between experimental data (re-elaborated from Villar (2004)) and model predictions on MX-80 bentonite compacted at four different dry densities. . . . . . . . . . . . . . . . . . .

13 Calibration of the water retention model against experimental data (Wang et al. 2013; Gatabin et al. 2016) on a MX-80 bentonite/sand mixture compacted at two different dry densities. Wetting path under confined conditions.

14 Comparison between experimental data (Wang et al. 2013; Gatabin et al. 2016) and model predictions on a MX-80 bentonite/sand mixture compacted at two different dry densities. Wetting path under unconfined conditions: (a) initial dry density $\rho_{d}=1.71 \mathrm{Mg} / \mathrm{m}^{3}$; (b) initial dry density $\rho_{d}=2.04 \mathrm{Mg} / \mathrm{m}^{3} \ldots \ldots \ldots \ldots \ldots \ldots$

15 Model predictions for the evolution upon wetting of the total, microstructural and macrostructural void ratios of a compacted MX-80 bentonite/sand mixture: (a) wetting under confined conditions; (b) wetting under unconfined conditions. . . . . . . . . . . . . . . . . 
Table 1: Parameters of the microstructure evolution law for three bentonite-based materials.

\begin{tabular}{cccc}
\hline Material & $\beta_{0}$ & $\beta_{1}$ & $e_{m 0}$ \\
\hline Febex & 0.15 & 0.25 & 0.35 \\
MX-80 & 0.48 & 0.1 & 0.31 \\
MX-80/sand mixture $(70 / 30)$ & 0.18 & 0.1 & 0.29 \\
\hline
\end{tabular}

Table 2: Parameters of the water retention model for three bentonite-based materials.

\begin{tabular}{cccccc}
\hline Material & \multicolumn{2}{c}{ Microstructure } & \multicolumn{3}{c}{ Macrostructure } \\
& $C_{a d s}$ & $n_{a d s}$ & $A$ & $n$ & $m$ \\
& $\left(\mathrm{MPa}^{-1}\right)$ & & $(\mathrm{MPa})$ & & \\
\hline Febex & 0.0028 & 0.78 & 0.24 & 3 & 0.15 \\
MX-80 & 0.0075 & 1.5 & 0.2 & 3 & 0.15 \\
MX-80/sand mixture & 0.0053 & 0.79 & 0.2 & 3 & 0.15 \\
\hline
\end{tabular}




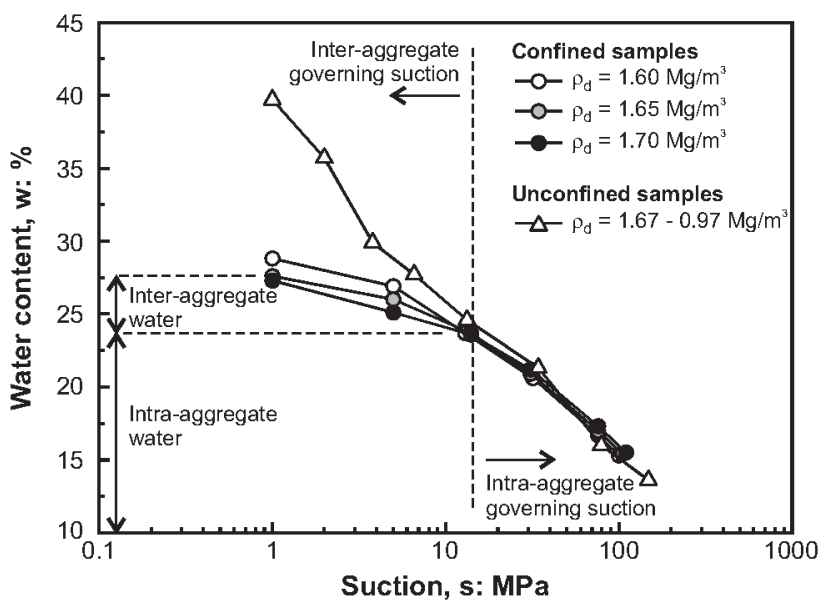

Figure 1: Water retention curves of compacted Febex bentonite obtained under confined and unconfined conditions (modified from Lloret et al. 2003).

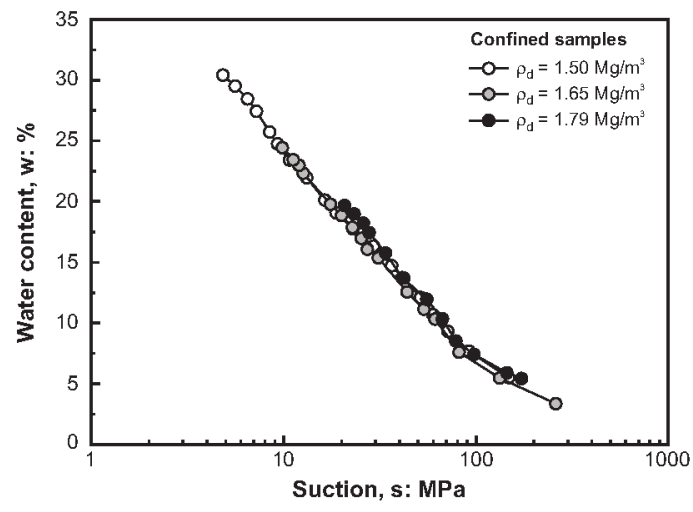

(a)

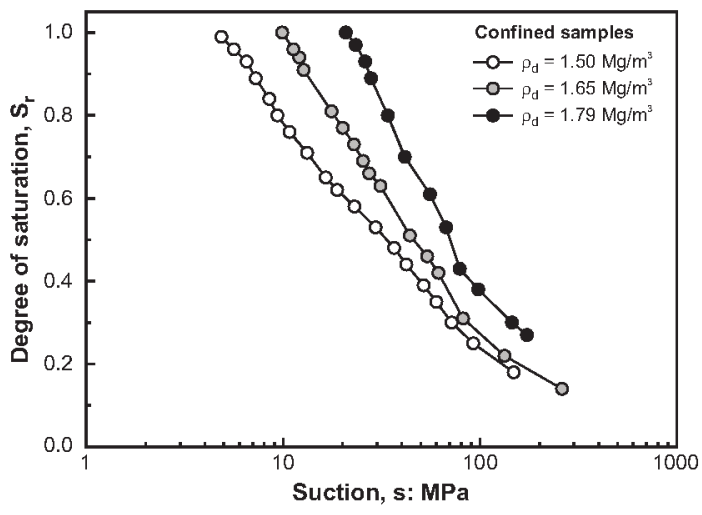

(b)

Figure 2: Water retention curves of MX-80 bentonite compacted at three different dry densities (Seiphoori et al. 2014): (a) water content versus suction; (b) degree of saturation versus suction.

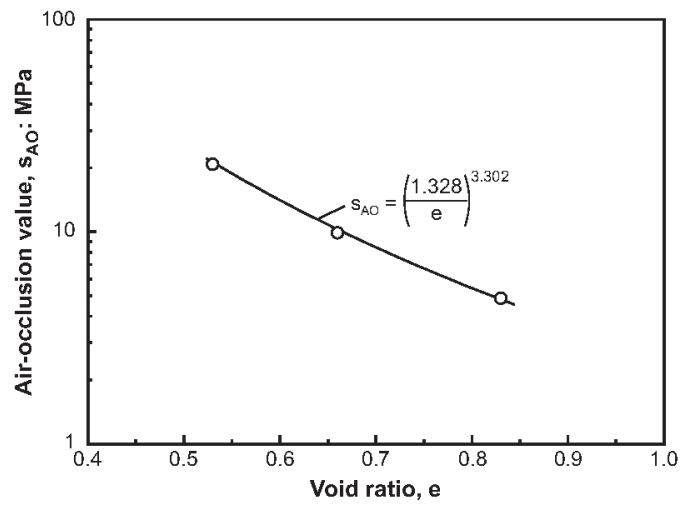

Figure 3: Evolution of the air-occlusion value of compacted MX-80 bentonite as a function of the void ratio (experimental data from Seiphoori et al. 2014). 


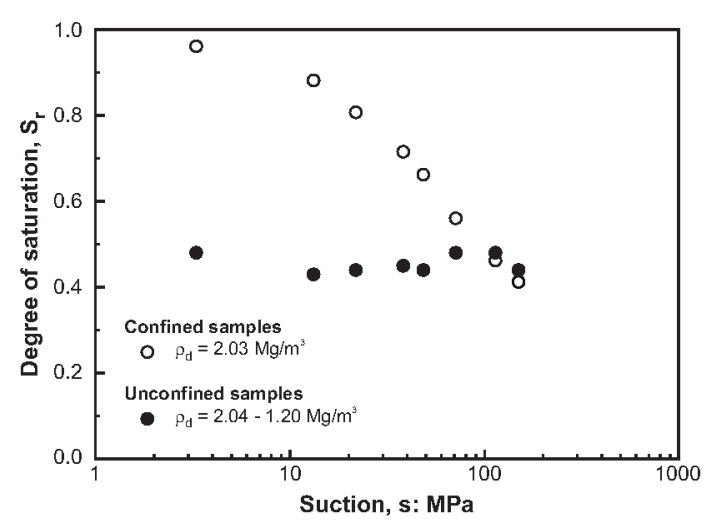

(a)

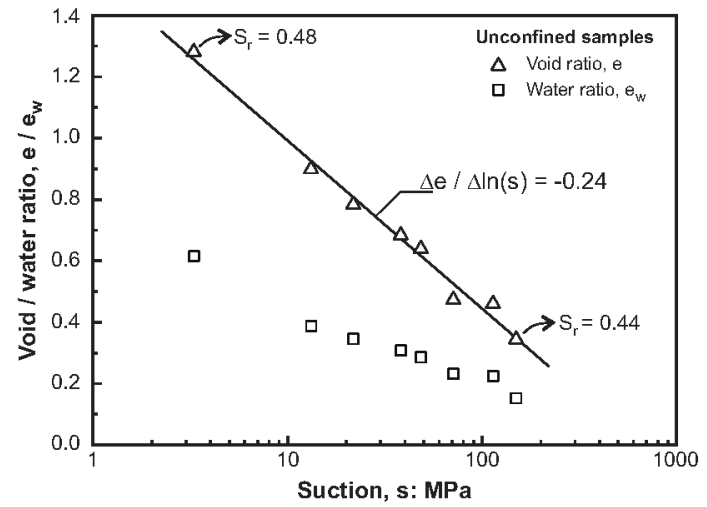

(b)

Figure 4: Water retention behaviour of a MX-80 bentonite/sand mixture compacted at a dry density $\rho_{d}=2.03 \mathrm{Mg} / \mathrm{m}^{3}$ (Gatabin et al. 2016): (a) water retention curves obtained under confined and unconfined conditions; (b) evolution of the water and void ratios upon wetting under unconfined conditions.

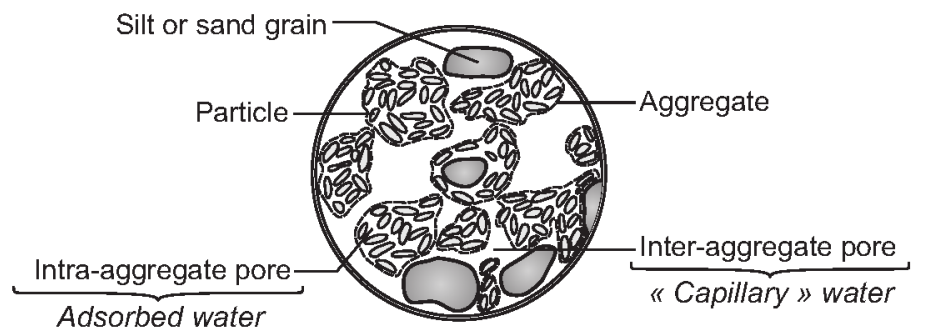

Figure 5: Conceptual representation of the water retention mechanisms in compacted bentonites.

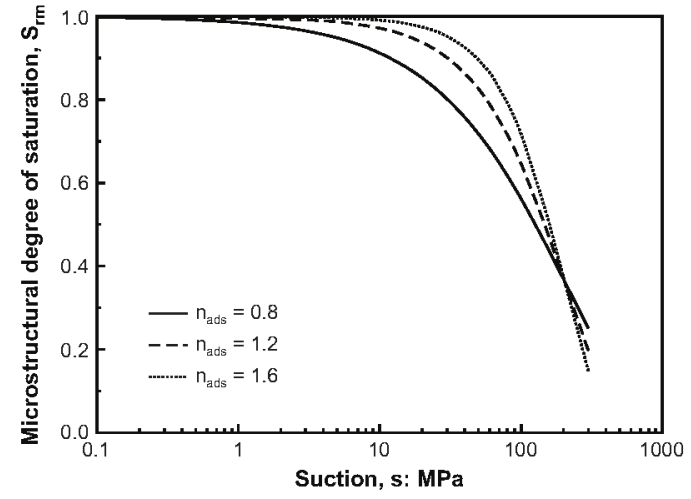

(a)

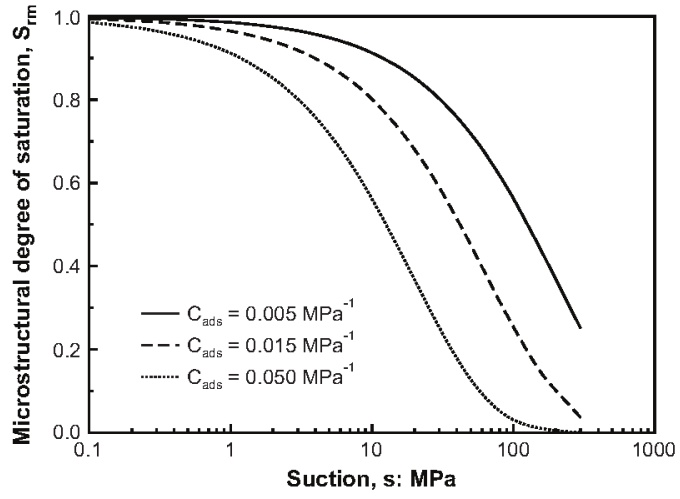

(b)

Figure 6: Influence of the microstructural parameters on the shape of the microstructural water retention curve $\left(e_{m}=0.3\right.$ is taken constant): (a) influence of $n_{a d s}\left(C_{a d s}=0.005\right.$ $\left.\mathrm{MPa}^{-1}\right)$; (b) influence of $C_{a d s}\left(n_{a d s}=0.8\right)$. 


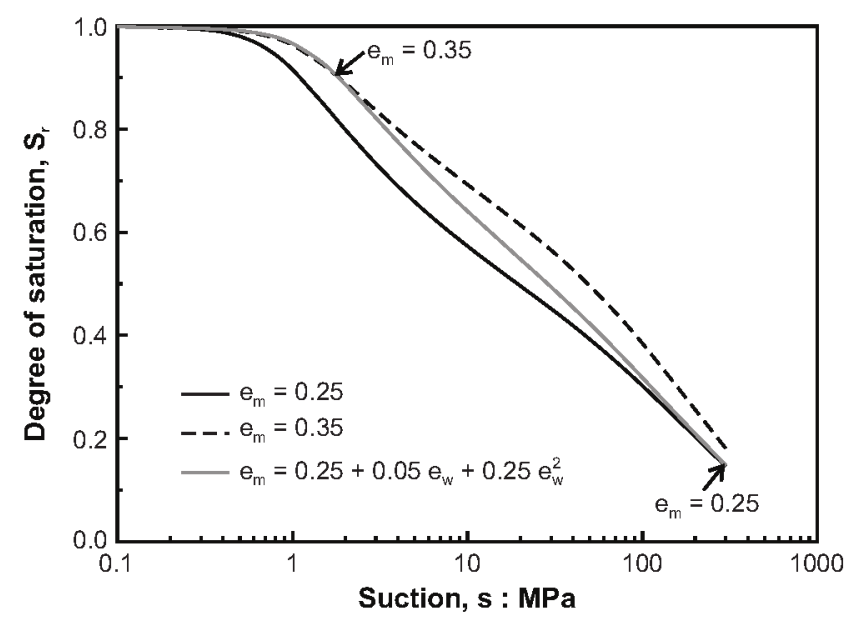

Figure 7: Influence of microstructure evolution on the shape of the (global) water retention curve. The parameters of the model are $C_{a d s}=0.005 \mathrm{MPa}^{-1}, n_{a d s}=0.8, e=0.6, A=0.3$ $\mathrm{MPa}, n=3$ and $m=0.15$.

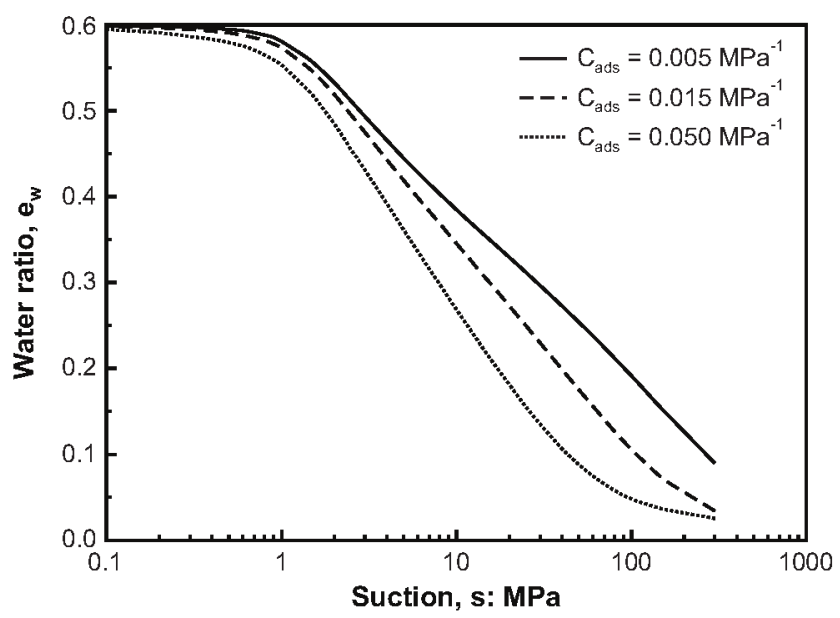

Figure 8: Influence of $C_{a d s}$ on the shape of the (global) water retention curve. The parameters of the model are $n_{a d s}=0.8, e=0.6, A=0.3 \mathrm{MPa}, n=3, m=0.15$, $\beta_{0}=0.25, \beta_{1}=0.05$ and $e_{m 0}=0.25$. 


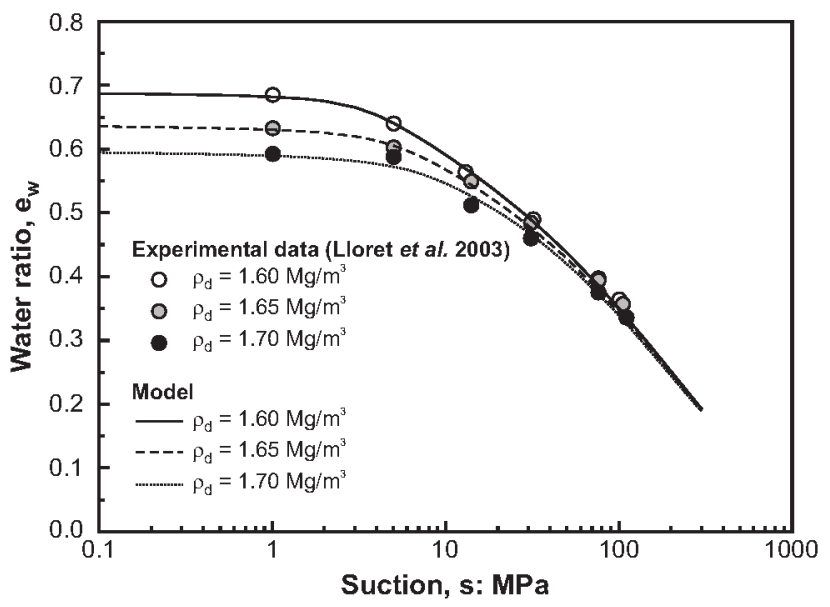

Figure 9: Calibration of the water retention model against experimental data (Lloret et al. 2003) on Febex bentonite compacted at three different dry densities. Wetting path under confined conditions.

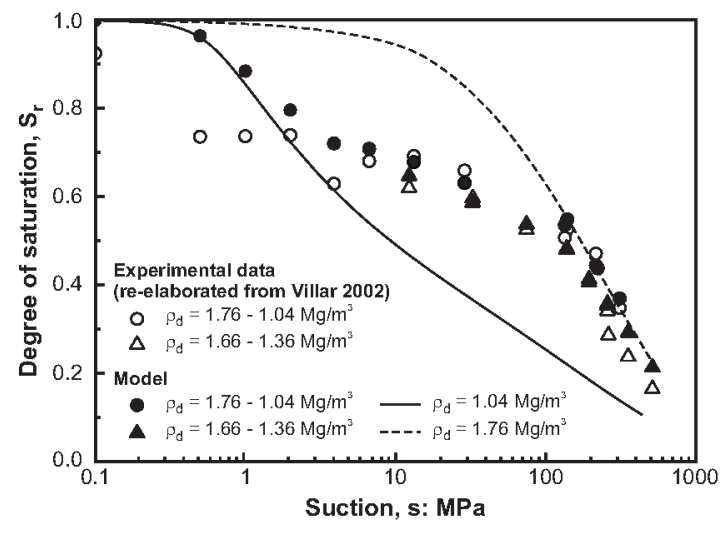

(a)

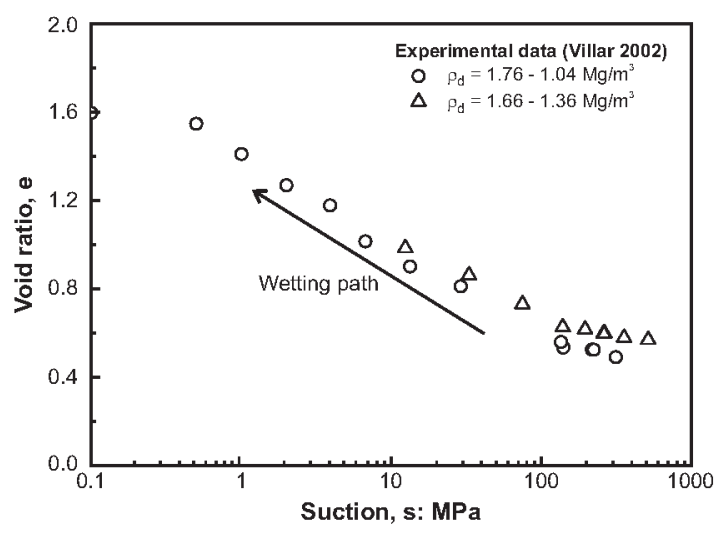

(b)

Figure 10: Comparison between experimental data (Villar 2002) and model predictions on compacted Febex bentonite. Wetting path under unconfined conditions: (a) degree of saturation; (b) void ratio (experimental). 


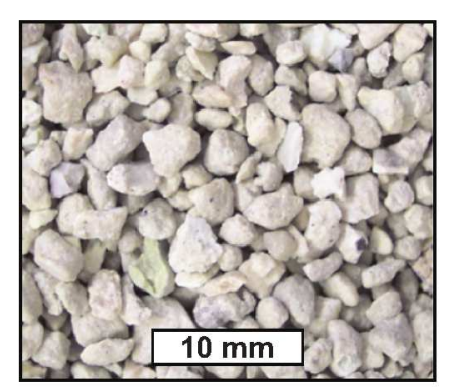

(a)

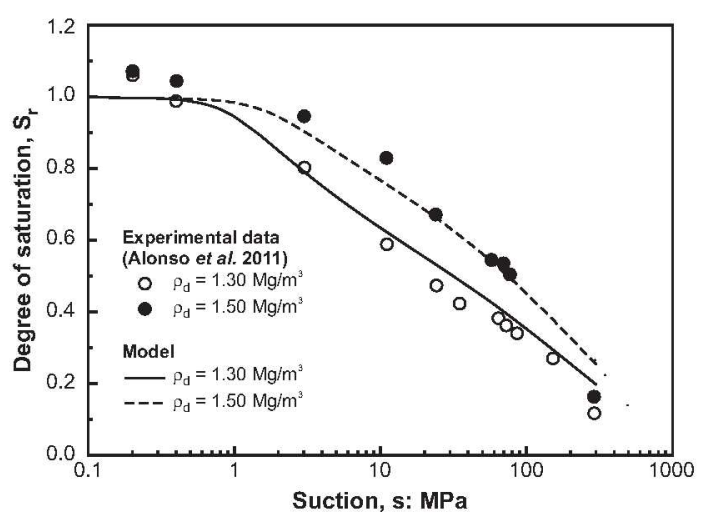

(b)

Figure 11: Water retention properties of granular Febex bentonite (Alonso et al. 2011). The maximum pellet size is $4 \mathrm{~mm}$. (a) Photograph of the granular mixture; (b) comparison between experimental data and model predictions.

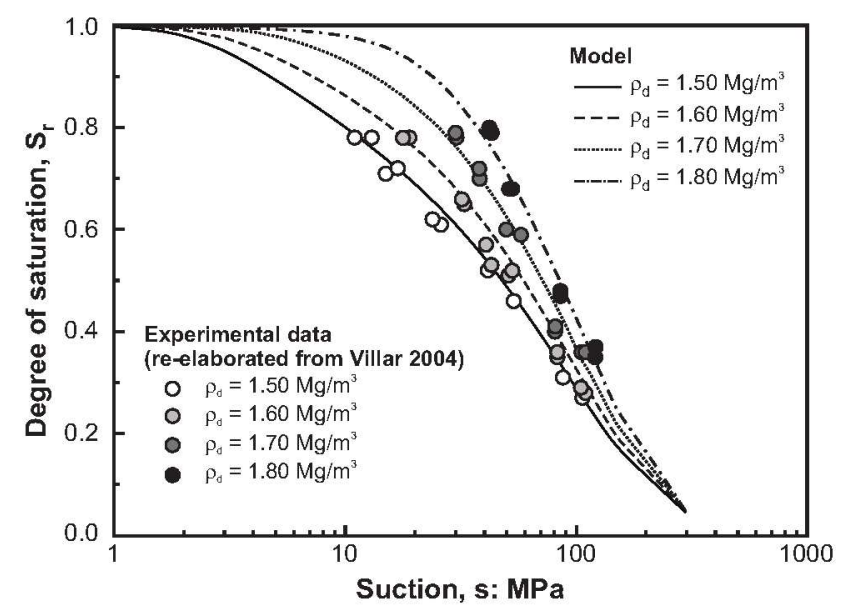

Figure 12: Comparison between experimental data (re-elaborated from Villar (2004)) and model predictions on MX-80 bentonite compacted at four different dry densities. 


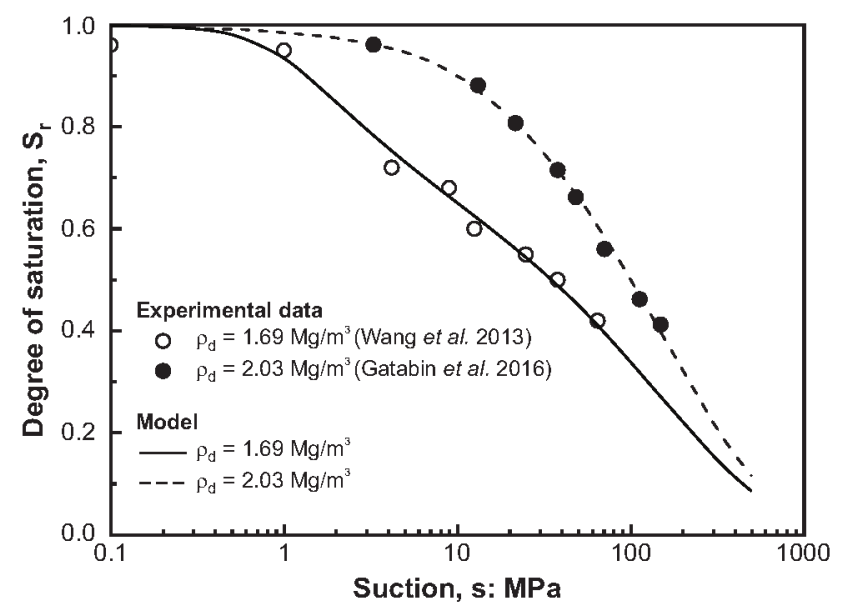

Figure 13: Calibration of the water retention model against experimental data (Wang et al. 2013; Gatabin et al. 2016) on a MX-80 bentonite/sand mixture compacted at two different dry densities. Wetting path under confined conditions.

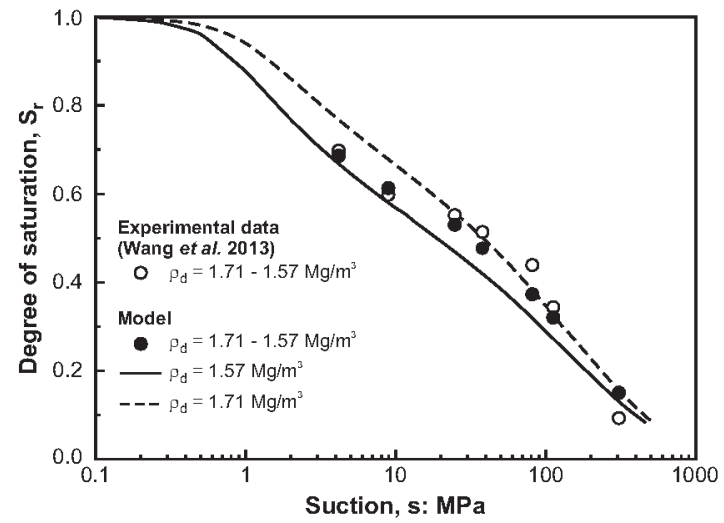

(a)

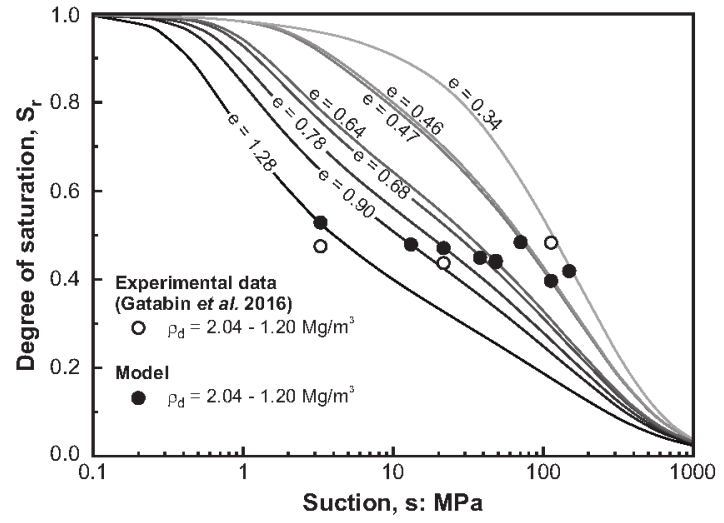

(b)

Figure 14: Comparison between experimental data (Wang et al. 2013; Gatabin et al. 2016) and model predictions on a MX-80 bentonite/sand mixture compacted at two different dry densities. Wetting path under unconfined conditions: (a) initial dry density $\rho_{d}=1.71$ $\mathrm{Mg} / \mathrm{m}^{3}$; (b) initial dry density $\rho_{d}=2.04 \mathrm{Mg} / \mathrm{m}^{3}$. 


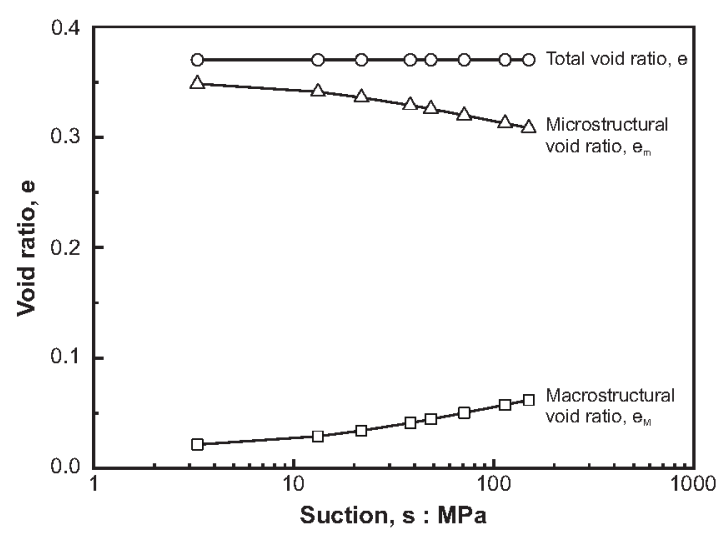

(a)

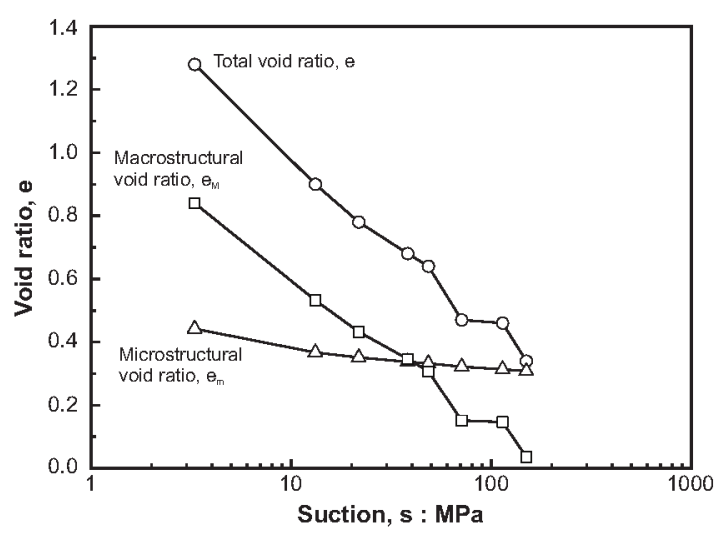

(b)

Figure 15: Model predictions for the evolution upon wetting of the total, microstructural and macrostructural void ratios of a compacted MX-80 bentonite/sand mixture: (a) wetting under confined conditions; (b) wetting under unconfined conditions. 